\title{
ULTRASSOM TERAPÊUTICO NO TRATAMENTO DA LESÃO MUSCULAR: REVISÃO SISTEMÁTICA
}

\section{THERAPEUTIC ULTRASOUND IN THE TREATMENT OF MUSCLE INJURY: SYSTEMATIC REVIEW}

\begin{abstract}
Maria Cecília Ribeiro Bruning*, Débora Pelegrini Silva**, Maria das Graças Anguera***, Gladson Ricardo Flor Bertolini****
\end{abstract}

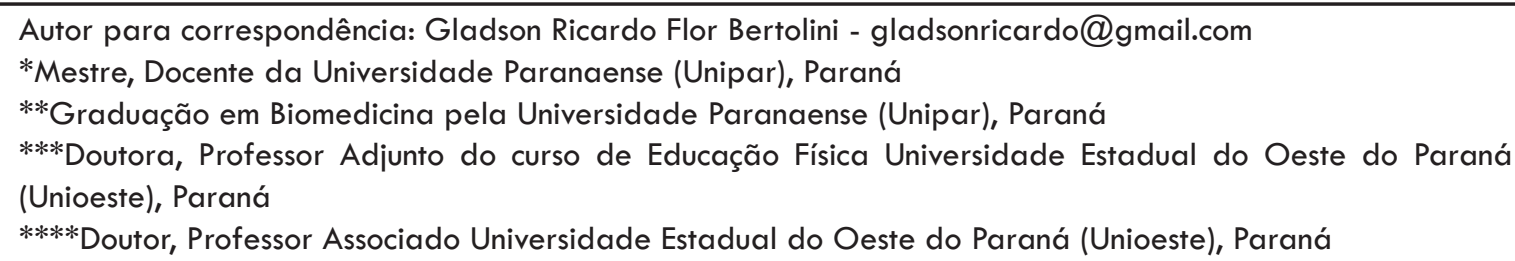

\section{R E S U M O}

\begin{abstract}
Introdução: As complicações decorrentes de lesões musculares diminuem a capacidade funcional podendo prejudicar o retorno às atividades rotineiras. $O$ uso do ultrassom como recurso no tratamento dessas lesões é frequente, porém, não há consenso científico na literatura em relação à efetividade desse recurso. Objetivo: analisar, por meio de revisão sistemática da literatura, os efeitos do ultrassom terapêutico em lesões musculares de animais e humanos. Metodologia: Trata-se de uma revisão sistemática, conduzida conforme a metodologia PRISMA. Foram incluídos artigos científicos com experimentos controlados, tanto em animais quanto em humanos, com os descritores "Terapia por ultrassom" e "ultrassom" associados com "músculo" (também traduzidos na língua inglesa) pesquisados nas bases de dados PubMed, Bireme e Scielo entre os anos de 2006 a 2013. Resultados: O tratamento de lesões musculares com ultrassom mostrou ser uma técnica eficaz em animais, no entanto, em humanos não apresentou diferença que demonstrasse a sua efetividade. Conclusão: apesar dos resultados conflitantes entre estudos em animais e em humanos, observaram-se resultados pró-reparo nos primeiros e ausência naqueles com humanos, contudo, são necessários mais estudos na área.
\end{abstract}

Palavras-Chave: Ultrassom; Terapia por ultrassom; Músculo esquelético. 
Introduction: The complications of muscle injuries can cause decrease in functional capacity and may impair the return to daily activities. The use of ultrasound as a resource in the treatment of these lesions is frequent, however, there is no scientific consensus in the literature regarding the effectiveness of this feature. Objective: To analyze, through a systematic review of the literature, the effects of therapeutic ultrasound in muscle injuries to animals and humans. Methodology: It is a systematic review, conducted according to the methodology prisma. Were included scientific articles with controlled experiments, both in animals and in humans, with the keywords "therapy by ultrasound" and "Ultrasound" associated with "muscle" (also translated in English) searched in the databases PubMed, Bireme and Scielo databases between the years of 2006 to 2013. Results: The treatment of muscle injuries with ultrasound has proven to be an effective technique in animals, however, in humans showed no difference to demonstrate its effectiveness. Conclusion: Despite the conflicting results between studies in animals and in humans, it was observed results pro-repaired in the first and absence among humans, however, more studies are needed in the area.

Key words: Ultrasound; Therapy for ultrasound; skeletal muscle. 


\section{INTRODUÇÃO}

As lesões musculares e os diferentes mecanismos que as causam, bem como o processo de reparo, apresentam um padrão constante, sendo que as alterações histológicas, nas diferentes fases do processo de reparo (destruição, reparo e remodelação), estão bem descritas na literatura'. Estas lesões são comuns em indivíduos que realizam intenso treinamento físico como soldados e atletas ${ }^{2}$, sendo que nestes, além de comuns, são responsáveis por extensos períodos de afastamento ${ }^{3}$, ainda podem apresentar uma cura lenta, sintomas persistentes e grande índice de recorrência, sendo as lesões posteriores geralmente mais graves do que a inicial ${ }^{4}$.

A partir da década de 1970 houve maior atenção com respeito ao uso de recursos fisioterapêuticos, como manipulações, programas de fortalecimento e recursos da eletrotermoterapia ${ }^{5}$, dente estes se pode citar o ultrassom terapêutico que é utilizado, há mais de 60 anos, em tratamentos de lesão muscular. É uma das técnicas terapêuticas de maior demanda dentro da fisioterapia ${ }^{6}$. É definido como uma onda sonora inaudível de alta frequência (acima de 20 $\mathrm{kHz}$ ), podendo causar elevação da temperatura e assim diversos efeitos fisiológicos, como aumento do fluxo sanguíneo, diminuição da dor e espasmo muscular, e aumento da extensibilidade do colágeno?. As frequências mais altas são utilizadas em tecidos mais superficiais e consequentemente as mais baixas são utilizadas para atingir tecidos mais profundos, em até $5 \mathrm{~cm}^{7}$. Também existem relatos sobre efeitos não térmicos advindos da vibração, que produziriam alterações nas concentrações de sódio e cálcio celular, e assim ações sobre o limiar de despolarização e ativação celular, com efeitos analgésicos e pró-reparo tecidual ${ }^{8}$.

Usualmente é aplicado, na forma pulsada, 24 horas após a lesão muscular, podendo levar ao aumento da síntese de proteínas contráteis, derivadas das células satélites das miofibrilas; estimular 0 realinhamento das fibras de colágeno; favorecer a formação de novas fibras musculares; e reduzir a área da lesão9-11. Contudo, na literatura não há consenso científico sobre o tratamento da lesão muscular $^{5}$, muito menos sobre a efetividade do ultrassom nesta ${ }^{12-14}$. Assim, o objetivo deste estudo foi realizar uma revisão sistemática sobre os efeitos do ultrassom terapêutico no tratamento de lesões musculares em animais e em humanos.

\section{MATERIAIS E MÉTODOS}

O presente estudo caracteriza-se como uma revisão sistemática, conduzida de acordo com a metodologia PRISMA (Preferred Reporting Items for Systematic Reviews and Meta-Analysis) ${ }^{15}$. Os artigos científicos sobre o tema foram pesquisados nas bases de dados PubMED, Google acadêmico, Scielo e buscas manuais.

Foram utilizados os seguintes descritores: "Ultrasound" e "Ultrasound Therapy", ainda, seus respectivos em português: "Terapia por ultrassom" e "ultrassom", associados com "muscle" e "músculo". Os termos, acima relacionados, foram encontrados como descritores nas bases: MeSH (Medical Subject Headings) e DeCS (Descritores em Ciências da Saúde).

Os resumos dos artigos encontrados foram analisados e selecionados pelos autores, conforme os critérios estabelecidos. Utilizaram-se como forma de inclusão os artigos escritos em inglês e português sobre a aplicação do ultrassom terapêutico como forma de tratamento para lesão muscular, sem associação com nenhum outro tipo de equipamento, técnica manual, ou qualquer outro tipo de intervenção e que dentro dos grupos experimentais houvesse um grupo controle. Sendo que as lesões musculares poderiam ser tanto lesões indiretas quanto diretas ${ }^{16}$.

Artigos não disponíveis na íntegra e/ou com ano de publicação superior a dez anos foram descartados (publicações abaixo do ano de 2005), bem como estudos que apresentaram a aplicação do ultrassom em músculo saudável. Posteriormente, elaborouse um fluxograma e as etapas organizadas em: identificação, seleção e elegibilidade. São apresentadas dois quadros, sendo um para estudos experimentais em animais (quadro 1) e outro, na 
qual foram realizados experimentos com humanos (quadro 2).

Utilizou-se como forma de inclusão os artigos escritos em inglês e português decorrentes sobre lesão muscular e o uso de ultrassom como única forma de tratamento, nos quais, pelo menos, um grupo estudado apresentou, efetivamente, a aplicação do ultrassom. Trabalhos experimentais de lesão em animais e com uso exclusivo do ultrassom para tratá-la foram inclusos nesta revisão e a comparação com o grupo controle ou grupo placebo também foi de estrita importância para seleção dos artigos.

\section{RESULTADOS}

Após as buscas, foram identificados 43 artigos disponíveis na íntegra (PubMed, Bireme e Scielo), sendo que destes, 06 foram excluídos por estarem duplicados, assim, 37 foram selecionados para leitura do resumo, após tal análise 30 artigos foram removidos por não se encaixarem na proposta da revisão sistemática, sendo selecionados 06 artigos ao final (Figura 1). A lista dos artigos selecionados foi revisada e incluída para análise dessa revisão sistemática (Quadro 1 - experimentos com animais e Quadro 2 - Estudos com humanos).

A amostra total dos artigos incluiu 130 animais e 28 humanos, tratados com ultrassom terapêutico de $1 \mathrm{MHz}$ ou de $3 \mathrm{MHz}$. Os animais foram submetidos a diferentes protocolos de lesões experimentais $(70$ animais foram submetidos à lesão por laceração, 40 animais submetidos à lesão por impacto, 20 animais submetidos à criolesão) e os humanos realizaram contração excêntrica máxima que simulava uma lesão.

Dentre os protocolos de tratamento destacam-se o ultrassom terapêutico no modo contínuo ou no modo pulsado, com uma média de seis sessões de aplicação, sendo o mínimo de cinco e o máximo de vinte minutos de aplicação, com doses mínimas de 30 $\mathrm{mW} / \mathrm{cm} 2$ e máximo de $1,5 \mathrm{~W} / \mathrm{cm} 2$. O período mais longo de avaliação do tratamento foi quatorze dias após a lesão. A publicação mais antiga, inserida

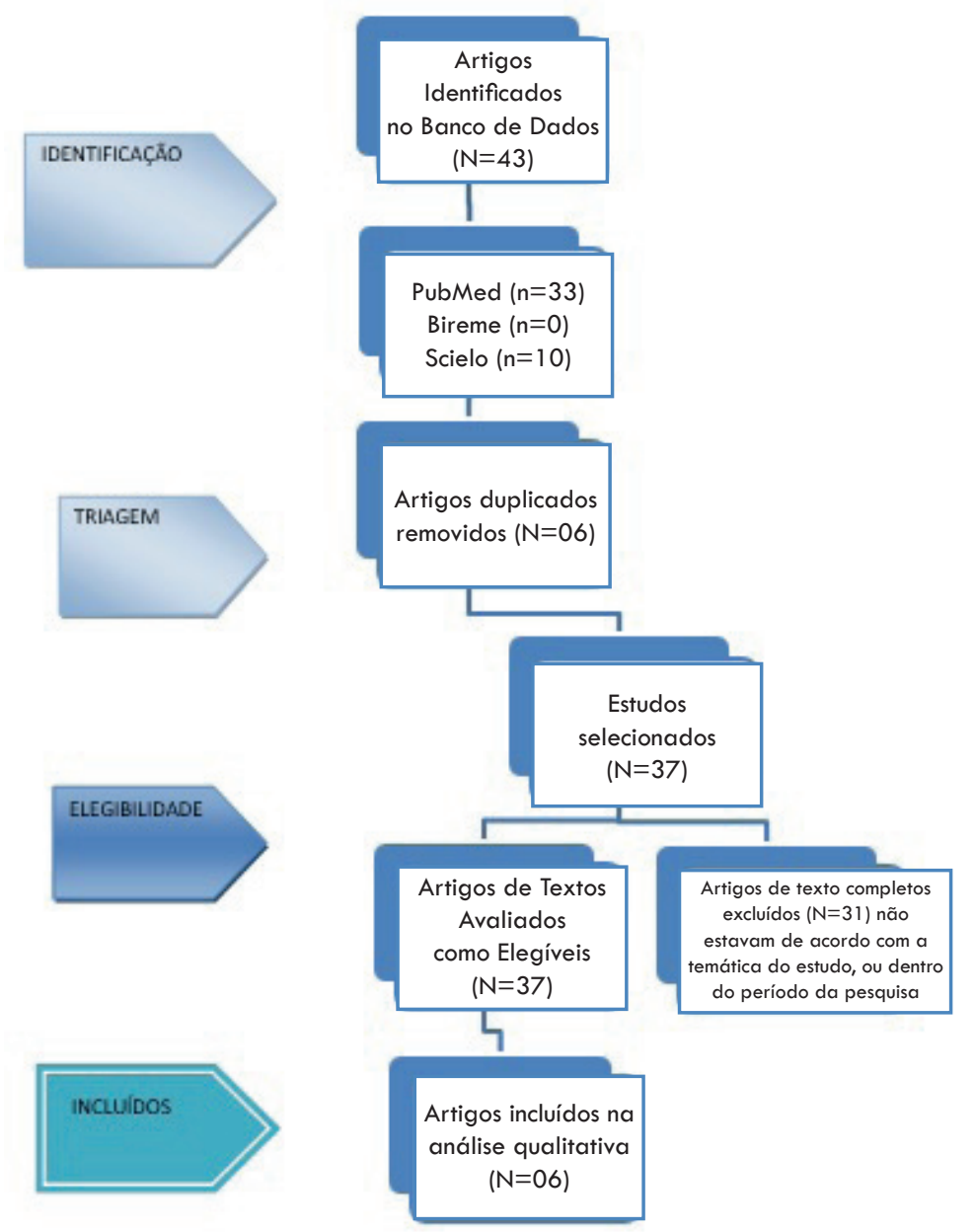

Figura 1. Fluxograma de identificação dos artigos de acordo com os critérios do PRISMA (2009). 
nesta revisão, foi de 2006 e o artigo mais atual de 2013. Os países de realização dos estudos incluíram Brasil, Índia, Estados Unidos e Taiwan.

Os protocolos de avaliação, utilizados nestes estudos, são validados pela literatura com a finalidade de analisar imunohistoquimicamente a presença de desmina e de colágeno no local da lesão, análise da secção transversa do músculo, da concentração de proteínas musculares no local da regeneração da lesão e contagem dos mionúcleos ${ }^{11-13}$.

Os efeitos do tratamento por ultrassom variaram

\section{DISCUSSÃO}

Em quatro estudos experimentais foi utilizado o músculo gastrocnêmio, em diferentes tipos de lesão como contusão, criolesão, lesão por impacto ou laceração $0^{911-13}$, devido sua localização e função, pois Matheus et al. ${ }^{9}$ afirmam que este trabalha sob condições de atividade física extrema e tem risco aumentado de lesão e rupturas; já nos estudos com humanos, Delgado-Diaz et al. ${ }^{17}$ utilizaram o vasto lateral e Shankar et al. ${ }^{14}$ os flexores de cotovelo por meio de lesão por contração excêntrica, .

Nos estudos com humanos, Delgado-Diaz et al. ${ }^{17}$ realizaram um protocolo de lesão muscular em 16 homens com idade entre 18-29 anos, por contração excêntrica máxima do músculo quadríceps, a avaliação ocorreu verificando-se creatina kinase e IGF-1, além da análise da força muscular pós lesão

Nos experimentos com animais houve maior número de miofibrilas no local da lesão no grupo que recebeu ultrassom, e evidência histológica de melhora precoce em relação ao grupo controle que apresentou maior tecido fibrótico e presença de macrófagos no local ${ }^{13}$; outro estudo a presentou maior carga de alongamento no limite máximo, e rigidez mais perto do grupo sem lesão para grupos tratados com ultrassom de 1 e $3 \mathrm{MHz}$ em relação ao grupo controle sem lesão; ainda, zona de regeneração com vascularização, mioblastos, desmina, deposição precoce e organização do colágeno ${ }^{11,12}$, maior expressão da COX-2, recrutamento precoce de de diferenciação das células o que favorece a regeneração ${ }^{12}$, regeneração das miofibrilas ${ }^{13}$, apresentação de uma estrutura tecidual mais organizada, melhora na expressão da COX211, melhora das propriedades mecânicas dos músculos lesionados", estes resultados demonstrados nos trabalhos experimentais em animais, já nos trabalhos com humanos não se encontrou, mudança na expressão de marcadores padrão relacionados a lesão muscular ${ }^{17}$, e não se encontrou influencia do ultrassom na recuperação quando avaliados os parâmetros da lesão ${ }^{14}$.

células inflamatórias e formação de miofibrilas ${ }^{11}$.

Tais efeitos, citados acima, mostram ativação tecidual pelo ultrassom, Vasquez et al. ${ }^{18}$, realizaram a aplicação do ultrassom terapêutico, contínuo e pulsado $(3 \mathrm{MHz}, 1 \mathrm{~W} / \mathrm{cm} 2,1$ minuto/sessão, 10 dias) sobre o gastrocnêmio saudável de ratos, e verificaram que não houve nenhum dano ou alteração histológica nas fibras musculares, ou tecido conectivo, nem foram observadas áreas de inflamação ou necrose. Porém encontraram maior diâmetro das fibras musculares nos grupos com aplicação de ultrassom continuo e pulsado, em comparação com o grupo controle por maior presença de células satélites no local. Bertolini et al. ${ }^{19}$ também observaram alterações no comprimento muscular de sóleos de ratos submetidos a ultrassom térmico ( $1 \mathrm{MHz}, 0,5$ e $1,0 \mathrm{~W} / \mathrm{cm} 2)$ associados à alongamento, porém, não houve alterações em comprimento e número de sarcômeros. A aplicação do ultrassom foi no músculo vasto lateral, 1,5W/ $\mathrm{cm} 2$, equipamento de $1 \mathrm{MHz}$ durante 10 minutos. Ao término do tratamento foi realizada a biópsia da região medial do músculo, para análise posterior, as avaliações mostraram aumento significativo da creatina kinase e uma diminuição de produção de força muscular local pós-lesão, quando comparado com o grupo controle. Os autores não encontraram alteração na expressão total de IGF-1 no grupo que sofreu a intervenção por ultrassom em comparação com o grupo controle sem lesão e o grupo lesão sem 
tratamento. Shankar et al. ${ }^{14}$ também realizaram protocolo de lesão por contração excêntrica, porém dos flexores de cotovelo, do lado não dominante, sendo a amostra composta de 12 homens, divididos entre grupo controle e grupo tratamento com ultrassom $0,8 \mathrm{~W} / \mathrm{cm}^{2}$. As avaliações foram realizadas com a Escala Visual de Dor, goniometria e Jonex Muscle Master Score. Ao final do experimento os autores não encontraram benefícios na aplicação do ultrassom.

Assim, observou-se que nos estudos em animais houve características de recuperação precoce com o uso de ultrassom, mas, não comprovado nos estudos com humanos, o que vai ao encontro ao relatado em revisão, sobre tratamentos para lesão muscular, de Järvinen et l. $^{20}$ que afirmam que apesar das evidências obtidas em estudos com animais na diminuição da dor e auxílio no estágio inicial de regeneração muscular, em humanos não há resultados significativos na recuperação precoce das lesões. Contudo, os dados apresentados aqui devem ser analisados com cautela, devido à heterogeneidade dos artigos (divisão dos grupos, número de animais e humanos por grupo, forma de lesão, dose e tempo de aplicação do ultrassom), entre outros fatores que podem aumentar os riscos de viés. Outro fato discutível é o uso isolado do ultrassom, pois quando associado à outra técnica, pode sofrer o efeito desta de modo a intervir em seu resultado. Vale destacar que nos artigos selecionados não houve relatos de efeitos adversos, tais como: queimaduras e intolerância, tornando o ultrassom uma terapia segura dentro dos parâmetros apresentados.

Apenas os trabalhos de Montalti et al. ${ }^{11}$ e Matheus et al. ${ }^{9}$, citam o calculo de SATA para definir as doses utilizadas no tratamento das lesões, sendo que nenhum dos artigos analisados cita qual foi a profundidade dessa lesão provocada por criolesão, laceração etc, nem nos trabalhos com humanos nem nos com animais essa informação é passada. Os tempos das sessões variaram entre o mínimo de 5 minutos $^{9,12}$, até o máximo de 20 minutos $^{11,13}$, e ○ número de sessões foi de uma ${ }^{17}$ até $14^{12}$, onde a quantidade de sessões não foi justificada nos artigos, bem como o porque da escolha do ultrassom de $1 \mathrm{MHZ}$ ou de $3 \mathrm{MHZ}$.

Otratamento comultrassomterapêuticonas disfunções musculares como opção complementar, muitas vezes é baseado em opiniões pessoais ou experiências clínicas, enfatizando assim a necessidade de futuras pesquisas, com metodologia bem fundamentada que comprovem a real efetividade desta intervenção especialmente devido a controvérsia que existe entre trabalhos realizados com animais que mostram bons resultados em comparação com humanos. Além de um tópico pouco explorado que é a calibração dos equipamentos, que pode produzir diferenças na real saída dos equipamentos e assim divergências nos resultados.

\section{CONCLUSÃO}

Diante dos artigos avaliados, houve melhora nos parâmetros relacionados à regeneração muscular com $\circ$ uso do ultrassom terapêutico apenas em protocolos aplicados em animais, mas, não houve efeitos pró-reparo em humanos, sem efeitos adversos em nenhuma das lesões avaliadas, portanto pode ser considerada uma intervenção segura. Contudo, devido ao pequeno número de manuscritos avaliados, observa-se a necessidade de mais estudos, principalmente ensaios clínicos, buscando uma possível validação do mesmo, como forma de tratamento em lesões do tecido muscular esquelético.

\section{REFERÊNCIAS}

1. Fisher BD, Hiller CM, Rennie SGA. A comparison of continuous ultrasound and pulsed ultrasound on soft tissue injury markers in the rat. J Phys Ther Sci. 2003;15(2):65-70. doi: 10.1589/ipts.15.65

2. Cameron KL, Owens BD. The burden and management of sports-related musculoskeletal injuries and conditions within the US military. Clin Sports Med. 2014;33(4):573-89. doi: 10.1016/i. csm.2014.06.004

3. Lee JC, Mitchell AWM, Healy JC. Imaging of muscle injury in the elite athlete. $\mathrm{Br} J$ Radiol. 201 2;85(1016):1 173-85. doi: 10.1259/ $\mathrm{bjr} / 84622172$ 
4. Heiderscheit BC, Sherry MA, Silder A, Chumanov ES, Thelen DG. Hamstring strain injuries: recommendations for diagnosis, rehabilitation and injury prevention. J Orthop Sports Phys Ther. 2010;40(2):67-81. doi: 10.2519/jospt.2010.3047

5. Hamilton B. Hamstring muscle strain injuries: what can we learn from history? $\mathrm{Br} J$ Sports Med. 2012;46(13):900-3. doi: 10.1136/ bisports-2012-090931

6. Shah SGS, Farrow A, Esnouf A. Availability and use of electrotherapy devices: A survey. International Journal of Therapy and Rehabilitation. 2007;14(6):260-4. doi: 10.12968/ ijtr.2007.1 4.6.23895

7. Hanks J, Levine D, Bockstahler B. Physical Agent Modalities in Physical Therapy and Rehabilitation of Small Animals. Vet Clin North Am Small Anim Pract. 2015;45(1):29-44. doi: 10.1016/i. crsm.2014.09.002

8. Martignano CCS, Silva LI, Meireles A, Rocha BP, Rosa CT, Bertollini GRF. Artigo original Avaliação do ultrassom sobre a hiperalgesia e o edema em joelhos de rato Wistar e interferências de um inibidor de opioides endógenos. Fisioter Bras. 2013;14(4):28993

9. Matheus JPC, Oliveira FB, Gomide LB, Milani JGPO, Volpon JB, Shimano AC. Efeitos do ultrasom terapêutico nas propriedades mecânicas do músculo esquelético após contusão. Rev Bras Fisioter. 2008; 1 2(3):241-7. doi: 10.1590/S141335552008000300013

10. Freitas LS, Freitas TP, Silveira PC, Rocha LG, Pinho RA, Streck EL. Effect of therapeutic pulsed ultrasound on parameters of oxidative stress in skeletal muscle after injury. Cell Biol Int. 2007;31:482-8. doi: 10.1016/i.cellbi.2006.11.015

11. Montalti CS, Souza NVCKL, Rodrigues NC, Fernandes KR, Toma RL, Renno ACM. Effects of lowintensity pulsed ultrasound on injured skeletal muscle. Braz J Phys Ther. 2013;17(4):343-50. doi: 10.1590/ S1413-35552012005000101

12. Piedade $M C B$, Galhardo $M S$, Battlehner $C N$, Ferreira MA, Caldini EG, de Toledo OM. Effect of ultrasound therapy on the repair of Gastrocnemius muscle injury in rats. Ultrasonics. 2008;48(5):403-1 1. doi: 10.1016/i.ultras.2008.01.009

13. Chan Y-S, Hsu K-Y, Kuo C-H, Lee S-D, Chen $\mathrm{S}-\mathrm{C}$, Chen W-J, et al. Using low-intensity pulsed ultrasound to improve muscle healing after laceration injury: an in vitro and in vivo study. Ultrasound Med Biol. 2010;36(5):743-51. doi: 10.1016/i. ultrasmedbio.2010.02.010

14. Shankar G, Sinha AG, Sandhu JS. Pulsed ultrasound does not affect recovery from Delayed Onset Muscle Soreness. Online J Heal Allied Scs. 2006;5(1):1-6.

15. Liberati A, Altman DG, Tetzlaff J, Mulrow C, Gøtzsche PC, loannidis JPA et al. The PRISMA statement for reporting systematic reviews and meta-analyses of studies that evaluate healthcare interventions : explanation and elaboration. BMJ. 2009;339:b2700. doi: 10.1136/bmj.b2700

16. Mueller-Wohlfahrt H-W, Haensel L, Mithoefer $\mathrm{K}$, Ekstrand J, English B, McNally S et al. Terminology and classification of muscle injuries in sport: the Munich consensus statement. $\mathrm{Br} J$ Sports Med. 2013;47(6):342-50. doi:10.1136/ bisports-2012-091448

17. Delgado-Diaz DC, Gordon BS, Dompier $T$, Burgess S, Dumke $C$, Mazoué $C$ et al. Therapeutic ultrasound affects IGF-1 splice variant expression in human skeletal muscle. Am J Sports Med. 2011 ;39(10):2233-41. doi: $10.1177 / 0363546511414857$

18. Vásquez B, Navarrete J, Farfán E, Cantín M. Effect of pulsed and continuous therapeutic ultrasound on healthy skeletal muscle in rats. Int J Clin Exp Pathol. 2014;7(2):779-83.

\section{Bertolini GRF, Barbieri CH, Mazzer N.} Longitudinal analysis of soleus muscles of rats submitted to passive stretching with previous use of therapeutic ultrasound. Rev Bras Med do Esporte. 2009;15(2):1 15-8. doi: 10.1590/S151786922009000200006

20. Järvinen $T A H$, Järvinen $M$, Kalimo $H$. Regeneration of injured skeletal muscle after the injury. Muscles Ligaments Tendons J. 2013;3(4):33745 